\title{
Returning to the scene of the trauma in PTSD treatment - why, how and when?
}

\author{
Hannah Murray $^{1 *}$, Christopher Merritt ${ }^{2}$ and Nick Grey ${ }^{3}$ \\ ${ }^{1}$ Traumatic Stress Service, South-West London and St Georges NHS Trust, London, UK \\ ${ }^{2}$ Institute of Psychiatry, Psychology and Neuroscience, King's College London, London, UK \\ ${ }^{3}$ Centre for Anxiety Disorders and Trauma, South London and Maudsley NHS Foundation Trust, \\ London, UK
}

Received 6 February 2015; Accepted 1 October 2015

\begin{abstract}
Returning to the scene of the trauma is often recommended as part of trauma-focused cognitive-behavioural therapies for post-traumatic stress disorder (PTSD). Many clinicians avoid site visits due to lack of confidence or practical constraints; however, recent research suggests this is a valuable part of treatment. This article summarizes a rationale for including the site visit as part of cognitive therapy for PTSD, as well as the main considerations about when to carry it out in treatment. A practical framework for planning and implementing site visits is described.
\end{abstract}

Key words: Cognitive behaviour therapy (CBT), post-traumatic stress disorder (PTSD), site visit, trauma, traumatic stress.

\section{Introduction}

Returning to the scene of the trauma during trauma-focused cognitive behavioural therapy (TF-CBT) for post-traumatic stress disorder (PTSD) can often be a valuable part of treatment. However, many therapists do not feel competent in carrying out site visits. In a recent training evaluation, CBT therapists who routinely treated PTSD were asked to rate their own competency in carrying out a site visit with a client. Only two (5\%) out of the 40 therapists rated themselves as having fully developed this competency (H. Murray, unpublished data). Under-utilization of exposure-based techniques in treatment of PTSD has been noted in other studies (e.g. Becker et al. 2004) and can lead to service users receiving interventions that are not maximally effective.

This article aims to promote the use of site visits in TF-CBT by providing a clear theoretical rationale for the technique (based predominantly around Ehlers \& Clark, 2000; Ehlers et al. 2003, 2005, 2013, 2014), and presenting the available evidence regarding their use. A practical framework, with illustrative case examples, is also included, to guide therapists in how to plan and implement an effective site visit.

\footnotetext{
*Author for correspondence: Dr H. Murray, Traumatic Stress Service, South-West London and St Georges NHS Trust, London, UK (email: hannah.murray@swlstg-tr.nhs.uk).
} 


\section{Why do a site visit?}

Returning to the scene of the trauma is recommended in some TF-CBT approaches to PTSD, including prolonged exposure treatments (e.g. Foa \& Rothbaum, 1998), and cognitive therapy (Ehlers \& Clark, 2000). Site visits are described as part of treatment in some TFCBT case studies (Tolin \& Foa, 1999; Jones \& Banks, 2007; Grey et al. 2009), but little research has considered the specific impact of returning to the scene of the trauma and no known studies have compared the efficacy of TF-CBT conducted with vs. without a site visit.

A recent study by Murray et al. (2015) gathered feedback from 25 service users who had undertaken site visits as part of TF-CBT. Overall, 23/25 of those surveyed reported the site visit was 'helpful' or 'very helpful', with the remaining two reporting it was 'neither helpful nor unhelpful'. Qualitative feedback supported these responses, with most service users endorsing positive experiences on the visit leading to a sense of "closure and moving on'. For example, one participant in the study likened the site visit to 'closing a big book I had been reading for a long time'.

Different CBT models of PTSD describe slightly different functions of the site visit. Prolonged exposure treatments for PTSD (such as Foa et al. 2007) involve exposure to trauma-related stimuli, for example using an exposure hierarchy which may include a return to the scene of the trauma. The rationale for site visits in this model is to extinguish the fear associated with the site through exposure. The method of conducting a site visit may also vary depending on the therapeutic model. For example, in prolonged exposure, the client would be required to stay at the site until the anxiety habituated and reduced.

This paper will primarily consider using the site visit in cognitive therapy for PTSD (CTPTSD; Ehlers \& Clark, 2000). This model considers the maintenance of PTSD as arising from the processing of the traumatic experience in a manner which leads to a sense of current threat. This occurs via negative appraisals of the traumatic event and/or the sequelae, for example 'the world is dangerous', 'the event was my fault' or 'I'll never recover'. The model also predicts that traumatic memories are poorly laid down in autobiographical memory and are therefore easily triggered and feel current, leading to an ongoing sense of threat.

Revisiting the scene of the trauma can therefore achieve various therapeutic tasks according to the cognitive model:

- 'Then versus now' processing or 'stimulus discrimination'. In CT-PTSD, clients are encouraged to note similarities and differences between their triggers of intrusive memories and the trauma, with the primary aim of helping them to distinguish the past from the present. On site visits, noticing differences between the scene of the trauma now compared to when the trauma occurred facilitates discrimination between 'now' and 'then', helping the event feel more in the past. For example, one participant in the study by Murray et al. (2015), commented: 'It was helpful to return after 14 years and find changes in the venue and to see how it has moved on and in doing so it has urged me to do the same'.

- Finding new information. On site visits, clients may find new information about the traumatic event that they had not previously accessed, which may help them to reevaluate problematic appraisals. For example, new information may help clients to 
re-appraise decisions they and others made at the time of the trauma, which may seem more understandable when viewed with a new perspective.

- Reconstructing the trauma. This occurs when the site visit is used to reconstruct, elaborate and fill in any gaps in the trauma memory, therefore making it easier to process.

- Behavioural experiments. Sometimes a site visit can be a behavioural experiment in itself, for example testing out whether something bad will happen on returning to the site. On other occasions, particular behavioural experiments can be planned to test out specific beliefs or predictions.

Service users have endorsed all of the functions listed above (Murray et al. 2015). Using a grounded theory analysis of feedback, it emerged that the site visit led to a sense of 'closure and moving on', which was facilitated by processes including 'filling in the gaps', 'different look and feel to the site', 'overcoming fear and avoidance' and 'learning from experiences'. Few, if any, negative experiences were reported, and were usually ameliorated by the presence of a supportive therapist and appropriate follow-up.

\section{When to do a site visit}

Although there has been no research indicating the optimal time to revisit the scene of a trauma, most therapists generally find it clinically appropriate in later stages of therapy. Within the Ehlers group's efficacy and effectiveness studies, in a typical 12 session CT-PTSD intervention, the site visit would be around session 9 (Ehlers et al. 2003, 2005, 2013, 2014). As visiting the site can provoke a strong emotional response, many therapists will first work on other therapy tasks, such as reliving the memory in imagination, before visiting the site. It is often used in the latter stages of therapy as a 'final hurdle' in elaborating and updating the trauma memory and testing out beliefs associated with the traumatic event.

In some circumstances, it can be useful to carry out site visits earlier in treatment. Some clients do not find the idea of returning to the trauma site particularly frightening or may already be visiting it (particularly if it occurred in their own home or workplace). It can be useful to visit the site earlier in treatment when clients have particular difficulty in accessing the trauma memory or the associated emotions. For example, if a client struggles to access the trauma memory during imaginal reliving, the therapist may try 'in vivo' reliving, by returning to the scene of the trauma to reconstruct the trauma memory.

Conducting a site visit may not always be possible or advisable in treatment. As discussed further below, some sites may be inaccessible or dangerous to visit, although the use of virtual or approximated visits may be a useful alternative. Some clients may feel too anxious about the possibility or experience high levels of dissociative symptoms which would be triggered by visiting the site. In such cases, site visits may be considered at a later stage in treatment, or beliefs about what may occur on the site visit tested out using a series of behavioural experiments.

\section{Planning a site visit}

Advance planning, in collaboration with the client, will help to maximize benefits and reduce any risks in returning to the scene of the trauma. 


\section{Engaging the client}

Many clients will feel anxious about revisiting the trauma site. As with other aspects of trauma therapy which have the potential to be distressing, the therapist needs to respect and validate the client's feelings, to understand the concerns underlying these, and also encourage and gently challenge them, even when a task is frightening or potentially upsetting.

A good place to start is explaining a rationale for a site visit. Although the terminology may be adapted, some concepts may have already been introduced in cognitive therapy, such as 'then versus now' discrimination to help place the event in the past, how reconstructing the memory (which may have already been started in timelines, reliving, narratives, etc.) can help process it, how finding out new information is important in how people think about and make sense of trauma, as well as using experiments to test things out. It may also be useful to reference evidence that feedback from clients suggests that most describe site visits as useful (Murray et al. 2015).

Using metaphors can help make an explanation more meaningful. For example, if the rationale for returning is being explained in terms of filling in gaps in the memory and looking for further information, a relevant metaphor may be a police or 'Crimewatch' reconstruction. These are done in order to jog the memory of witnesses and to look for clues, just as you might when returning to a trauma scene.

Some beliefs regarding returning to the site may need to be addressed prior to the visit. For example, some clients overestimate the likelihood that something bad will happen again if they return to the site. Looking at the realistic risk of the client's feared outcome may be useful. The idea that PTSD heightens threat perception, and cognitive restructuring of appraisals of exaggerated likelihood of further trauma, may have already been covered in therapy, and need revisiting. The site visit can be planned as a behavioural experiment to test whether feared predictions occur. Other common fears, for example that anxiety will be overwhelming, can be addressed using standard cognitive restructuring techniques and behavioural experiments. Preparing for potential problems (see below) may also alleviate anxiety.

Taking a graded approach to returning to the site of the trauma is another option for highly anxious clients. This may include behavioural experiments, for example visiting another anxiety-provoking place, or somewhere near the scene of the trauma. Approximations of the site visit such as a virtual visit using Google Street View or Google Earth (see 'alternative site visits' section) and utilizing stimulus discrimination may help to build up to visiting the site.

\section{Setting goals and planning experiments}

Discussing in advance what the client hopes to get out of the visit will help with planning and managing the site visit. Goals will often include one or more of the functions previously described, and clients may have specific behavioural experiments or information-gathering exercises that require forward planning, for example meeting with a member of staff. However, agreeing some flexibility in the goals is also useful, as there are some aspects of the visit which will be unpredictable.

John was returning to a train station where he was mugged at knifepoint. He believed he was mugged because he looked 'like a victim', and something about the way he had behaved that day had led to the mugging. 
John and his therapist decided to test this belief out when they went back to the station. They planned to sit near the station and watch people coming out to see how they behaved and whether there were any muggings. They also planned to ask any security staff at the station about safety in the station, how often muggings happened, and if they knew of any patterns in who was mugged. Lastly, they agreed that the therapist would record a video on John's phone of him walking out of the station in the way he had on the day of the mugging, so that they could watch it back in their next session and try to identify anything objective about how John had looked or behaved that may have made him vulnerable to being attacked.

\section{Practicalities}

Prior to the site visit, some practical arrangements need to be agreed with the client. These include agreeing a time and place to meet, a contingency plan for if either party is delayed and making a plan for potential problems. It is usually better to meet a short distance from the actual site of the trauma. Otherwise, if the client arrives before the therapist, it may be distressing for them to wait alone at the scene of the traumatic event. In some cases it is helpful to track the journey taken on the day of the trauma (while utilising stimulus discrimination) which sets the scene and context to begin the process of reconstructing the memory.

It is also important to leave sufficient time for the visit, including travel, debriefing time for after the visit and extra time to stay with the client in case they are distressed. A plan for after the visit is needed, for example going back to the office for a 'debrief', planning another session to discuss the visit, how the client will get home, and what they plan to do after the visit.

Therapeutic boundaries can feel different outside the therapy room and this may be helpful to discuss with the client. Sometimes a metaphor may be useful, for example likening the therapeutic boundary as a frame which client and therapist fit within and which is now being taken out of the office. The frame remains in place on the site visit, but consider how this will work in practice. For example, if you are travelling to the site together, would the client prefer you to just sit quietly, make small talk or discuss the visit?

Some visits require special arrangements, such as contacting the venue in advance to arrange the visit. Many places will be willing to help if the reasons for visiting are explained, as agreed with the client.

On a visit back to an intensive care unit, the therapist contacted the hospital to request access to the ward. A nurse agreed to meet them to show them the ward and to talk to Al.

Al had some beliefs about the traumatic experiences he had while on the ward. He had experienced terrifying hallucinations that people were trying to kill him and believed that this meant he was 'crazy'. Al also had some confused memories about invasive medical procedures and wasn't sure which of his memories were real and which were hallucinated.

On the visit, the nurse met with $\mathrm{Al}$ and answered his questions. She explained that many patients experience hallucinations. With Al's permission, she accessed his medical records and gave some details about the treatment he had received. Walking around the ward, Al was surprised at how different it looked from his memories. He saw staff treating other patients with kindness and respect, which helped update his memory of the nurses trying to harm him. 


\section{Planning for potential problems}

With all clients, it is important to consider and plan for potential problems. Depending on the type of visit, these might include practical issues (e.g. seeing someone the client knows), risk issues (e.g. seeing the perpetrator) or psychological issues (e.g. having a panic attack). Although it is impossible to plan for every contingency, some problems are preventable. Trouble-shooting can also help the experience feel more manageable for the client.

Clients sometimes worry about becoming very distressed or panicking on the site visit. Although this happens less often than people predict, it can be helpful to plan for this eventuality. By this stage of therapy, the therapist should have some knowledge of how to help the client in this scenario, and ask the client to identify coping strategies such as use of stimulus discrimination, which can be written on flashcards if needed. Ideally, if the client is panicking or feeling very distressed, the therapist should encourage staying at the site and help them to manage their panicky thoughts and feelings. Sometimes it is useful to temporarily move away from the trauma site to a pre-arranged 'safe place' (e.g. sitting in the car, a nearby coffee shop) to re-gather before continuing the visit if possible.

Similarly, if a client is prone to dissociation, grounding strategies should be prepared and practised in advance, which the therapist can prompt if required. If dissociation occurs unexpectedly, the therapist can use basic strategies such as calling the client's name, reminding them where they are and that they are safe and helping them to focus attention externally, preferably on something which was not present at the traumatic event, such as the therapist. It may be useful to take a map on the visit, to help the client to maintain an observer perspective. The therapist can redirect the client's attention to the map if they are beginning to dissociate.

Another contingency to plan for is seeing someone the client knows on the visit, particularly if the visit is in their home area, work environment or somewhere they previously lived. Clients can decide how they want to handle this situation, and can usually take the lead in such a scenario. Some people are happy to say 'this is my psychologist', but others may prefer to prepare an alternative explanation.

\section{Risk}

Most site visits will pose very few risks, but risk assessment is an essential part of planning the site visit. This includes following service lone working policies, including leaving the address of the visit, an expected return time, and a plan to be followed if this time is missed. If possible, two clinicians should attend a site visit, especially if potential risks have been identified. This enables one clinician to focus on the client, while the other monitors and deals with any risks.

Depending on the site, idiosyncratic risks may require consideration. In some cases a visit may be inappropriate due to risks, for example if a perpetrator continues to pose a risk to the client and is working or living at the location. Other risks may include re-visiting the site of a car accident which occurred on a motorway, where it would be dangerous to stop the car or walk around on the hard shoulder. With this example, it may still be possible to drive past the site and stop at the nearest safe place.

As clients with PTSD often overestimate risk, it can be difficult to assess clearly, so a judgement needs to be made based on the facts available. Clients can seek an unbiased 
perspective on risk by asking others, such as friends, family or others in their community. They can also try to recall how they would have perceived the risk prior to the trauma. Therapists can do some research to establish objective risk, such as visiting the area with a colleague prior to the site visit. Crime risk of a particular area can often be evaluated using various sources including crime statistics or by asking local police or community support officers. Some risk factors will depend on environmental factors which can be managed. For example, some places are safer to visit during daylight, or when there are more people around.

A risk plan should be formulated, including leaving the scene if risks emerge, and contacting the police. The therapist should agree that they will take the lead if a risk emerges and the client should be discouraged from stepping in.

Colin was planning to visit a caravan park where his brother had been murdered 20 years previously. He had not returned to the area since. The perpetrators of the murder had not lived in the area, so Colin thought it unlikely that he would see anyone related to the attack. Colin and his therapist researched the caravan park online to find out whether there were any potential risk factors. They discovered that it had now closed down and was due for demolition. With his permission, Colin's therapist contacted the owners of the park and requested access to the site. The owners agreed, and also confirmed that there had been no recent security incidents and there was a security office on site. Colin and his therapist 'checked in' with security on arrival and agreed a safety plan for while they were on site.

\section{What to do when you are there}

Overall, the therapist's general priorities are to support the client through the experience, provide positive feedback and encouragement and manage any problems or unexpected events. Clients will differ in how much they want or need prompting and guidance from the therapist. Taking regular ratings of how distressed a client is feeling, for example using Subjective Units of Distress Scale (SUDS) ratings out of 10, will give a rough indication of how the client is managing. Unlike prolonged exposure models, the use of SUDS ratings is only to 'take the emotional temperature' during the visit, not as a measure of habituation where the aim would be for SUDS ratings to reduce to a certain level before the visit is completed. Other aspects of the visit will differ slightly depending on the goals.

The therapist should be aware of any safety behaviours or avoidance, and gently encourage the client to engage with the experience, for example by having the client focus on sensory details, and ask questions to help clients observe details of the scene. Any observed avoidance may be relevant to the client's formulation, and to future work, including behavioural experiments.

As described by Ehlers \& Clark (2000) several therapeutic tasks may be undertaken on the visit:

- Reconstructing the trauma. With the client, 'walking through' the traumatic event at the site can help fill any memory gaps, and potentially reveal new information to update the memory.

- Noticing differences. To aid then/now discrimination, clients can be cued to notice differences between the appearance of the site now, and how it was at the time of the 
trauma to aid the experience of the trauma being over and in the past. For example, there have often been physical changes to a site such as building works, and memory triggers which are no longer present, e.g. ambulances. This also aids the creation of a strong alternative memory to 'compete' with the existing memory. This can be enhanced by doing something incongruous with the trauma at the site, for example moving around vigorously when a trauma involved being trapped or immobilized. The therapist can help the client to focus their attention on differences during the visit. This is in contrast to exposure models, where similarities would be the primary focus to aid habituation.

- Looking for new information. Visiting the site often allows a new understanding of aspects of the traumatic event. Certain information-gathering tasks may have been pre-planned, while other opportunities may emerge spontaneously.

On a return to the scene of a car accident, Aisha saw a parked lorry similar to the one which had struck her car. She initially felt extremely anxious but, with encouragement from her therapist, was able to go and examine the lorry. The driver came to drive away and Aisha told him about her accident. The driver was very sympathetic, explained about the difficulties of visibility in lorries and invited her into the cab to see what the driver of the lorry which had hit her would have been able to see. Although unplanned, the experience gave Aisha a different perspective on her accident, including allowing her to imagine what the accident had been like for the other driver.

- Behavioural experiments. Again, these may have been planned in advance, or the therapist may see opportunities to test beliefs while on the site visit. Therapists should be aware of safety behaviours the client is using on the visit and encourage the client to test out dropping them.

Danny was returning to a betting shop where he had witnessed an armed robbery. While in the shop, the therapist noticed Danny watching everyone coming in very closely and was reluctant to move away from the door. When asked, Danny realized that he was checking customers for visible weapons and making sure of their exit to keep himself and the therapist safe from another robbery. He and the therapist discussed the probability of another armed robbery happening that day and Danny concluded that the chances were low. He agreed to engage in a series of behavioural experiments, moving away from the door, turning his back to incoming customers and, eventually, closing his eyes for 2 minutes, to test whether it was safe to drop his guard.

Taking a photo at the scene for the client to keep is a useful memento, and helps to reinforce some of the helpful functions of the visit, providing a reminder of their achievement in returning to the site, a visual cue to then/now discrimination and helping to strengthen the alternative memory created by revisiting the site (Murray et al. 2015). One client in the study commented: 'in the new photos there are no evidence for the past, no big crowd, no police vehicles, fire brigades [sic]'.

If equipment is available and the client agrees, it can also be useful to film parts of the visit, or to audio record interviews. Therapists should be aware that certain places may have restrictions on photography and filming, and the privacy of others should be respected. If so, a photo can be taken in a slightly different location (for example, outside the hospital rather than on a ward). 
The point at which to end the site visit can be mutually agreed between the therapist and client. Unlike exposure models, this would not be decided by a particular reduction in anxiety levels (although this may well have occurred), rather at a point when the client and therapist feel the tasks of the site visit, such as reconstructing the event, stimulus discrimination, behavioural experiments and so on have been completed, and the maximum learning has been achieved.

\section{Managing unexpected events}

Although many possibilities can be considered when planning a site visit, it is impossible to predict every eventuality. When an unexpected situation arises, the therapist will need to use common sense to manage as best as possible. Risk protocols should be followed as appropriate. If the client is distressed, helping them to use coping strategies, such as grounding, cuing them to then/now discrimination and, if needed, moving away from the site to calm down and discuss whatever has happened may be useful.

Unexpected events can sometimes be useful therapeutically and some events that may initially seem problematic can be processed in a helpful way. The therapist can guide the client to think through the event afterwards.

Returning to the site where her boyfriend died in a motorcycle accident, Carrie witnessed a minor accident where two cars collided. Carrie felt very frightened and as if someone would be killed.

Talking it through afterwards with her therapist, Carrie concluded that the likelihood of someone dying in that type of accident was low. She also realized that she had coped well, having gone over to the drivers to check if they were okay and whether an ambulance was needed. This made her feel that she would be able to cope in the face of difficult situations in the future. She also noticed rescue factors which had been present, including the therapist, a passer-by who offered help, and the emergency services.

Carrie also realized that the event showed that it was a dangerous area of road with poor visibility. This helped her to feel less angry about the driver who had knocked her boyfriend off his bike and she decided to write to the council to alert them to the safety issue on that junction.

\section{Alternative site visits}

Sometimes it is not possible to return to the scene of the trauma, particularly if it occurred in another country or is too dangerous for the client. In these situations, alternative site visits can still facilitate the useful processes already described. Another use of alternative site visits is for clients who are too anxious to do a site visit immediately, as part of a graded approach to revisiting the scene.

One option is a 'virtual' site visit, where the therapist and client use digital materials to approximate returning to the scene of the trauma. Online resources such as Google Earth or Street View now give clear images of locations all over the world. Photographs and film footage can also be found online of many locations. For example, on the Chelsea and Westminster Hospital website there are short videos available about different wards, which can be used to approximate site visits to hospital settings (http://www.chelwest.nhs.uk/your-visit/patient-information-videos). YouTube is also 
a potential source of video material, but should be vetted by the therapist prior to sharing with the client.

In some cases, it may only be possible to return to a nearby location, or a location which has many matching features to the scene of the trauma.

Malcolm had experienced traumatic events in prison. He was unable to visit the prison grounds, but went with his therapist to the gates of the prison and walked around its periphery. He and his therapist took a picture of themselves at the gate of the prison.

He and his therapist also used Google Earth to obtain a satellite image of the prison grounds and Malcolm found an article about the prison on the internet, which had some photos from inside his block. This helped him to reconstruct the trauma memory with his therapist and discriminate how he remembered the prison looking from how it looked in the photos.

If the client is reluctant to return to the site, one option is for the therapist to go alone and take photos or videos to show the client. In some cases, the client may agree to come close to the site and the therapist can go and take some pictures or video to demonstrate to the client how the site appears that day. This sometimes encourages the client to build up to a full site visit.

If possible, the therapist should accompany the client on the site visit. If this is impossible, but the client is able to visit the site with another supportive individual, the therapist can guide the client and companion to carry out all the planning stages and collect their feedback afterwards.

In some cases, revisiting the scene of the trauma may be planned for after treatment ends as a follow-up goal, for example in the case of a survivor of the 2004 tsunami planning to return to Thailand in the future.

\section{Getting feedback and taking it forward}

After the site visit, feedback and discussion will help maximize learning experiences. If time does not allow returning to the office for a full debrief after the visit, some immediate feedback can be gathered by withdrawing to a quiet place. This will also enable the therapist to check how the client is feeling, and ensure that they can get home safely. On the rare occasion that a client has become very distressed or panicky on the site visit, the therapist can help them to calm down and prompt coping strategies.

Depending on how the visit went, and what the client learnt, there may be several ways to take the site visit forward. For example, if the client learnt new information about the trauma, this can be fed back into updating the trauma memory, or used to challenge beliefs. Video or audio recordings of the visit can be reviewed as homework to identify learning points. If the main goal of the visit was for the area near the trauma site to be perceived as less dangerous, further homework may involve reducing avoidance of the area.

\section{Summary}

The majority of clients find returning to the scene of the trauma to be a useful part of TF-CBT, and models, such as Ehlers \& Clark's CT-PTSD, explain how a visit might benefit clients through a number of different processes. Therapists need to plan carefully, in collaboration 
with clients, to maximize the benefits of the visit within a wider course of CT-PTSD or other TF-CBTs, and to assess and manage potential risks.

\section{Acknowledgements}

The authors thank Sharif El-Leithy for his advice and contributions to this paper and to all the service users who provided feedback on their site visits. Nick Grey receives salary support from the National Institute for Health Research (NIHR) Mental Health Biomedical Research Centre at South London and Maudsley NHS Foundation Trust and King's College London. The views expressed are those of the authors and not necessarily those of the NHS, the NIHR or the Department of Health.

\section{Declaration of Interest}

None.

\section{Recommended follow-up reading}

Ehlers A, Clark DM, Hackmann A, McManus F, Fennell M (2005). Cognitive therapy for posttraumatic stress disorder: development and evaluation. Behaviour Research and Therapy 43, 413431.

Grey N, McManus F, Hackmann A, Clark DM, Ehlers A (2009). Intensive cognitive therapy for post-traumatic stress disorder: case studies. In: A Casebook of Cognitive Therapy for Traumatic Stress Reactions (ed. N. Grey), pp. 111-130. Hove: Routledge.

Murray H, Merritt C, Grey N (2015). Clients' experiences of returning to the trauma site during PTSD treatment: an exploratory study. Behavioural and Cognitive Psychotherapy 43, 1-11.

\section{References}

Becker CB, Zayfert C, Anderson E (2004). A survey of psychologists' attitudes towards and utilization of exposure therapy for PTSD. Behaviour Research and Therapy 42, 277-292.

Ehlers A, Clark DM (2000). A cognitive model of posttraumatic stress disorder. Behaviour Research and Therapy 38, 319-345.

Ehlers A, Clark DM, Hackmann A, McManus F, Fennell M, Herbert C, Mayou R (2003). A randomized controlled trial of cognitive therapy, a self-help booklet, and repeated assessments as early interventions for PTSD. Archives of General Psychiatry 60, 1024-1032.

Ehlers A, Clark DM, Hackmann A, McManus F, Fennell M (2005). Cognitive therapy for posttraumatic stress disorder: development and evaluation. Behaviour Research and Therapy 43, 413431.

Ehlers A, Grey N, Wild J, Stott R, Liness S, Deale A, Handley R, Albert I, Cullen D, Hackmann A, Manley J, McManus F, Brady F, Salkovskis P, Clark DM (2013). Implementation of cognitive therapy for PTSD in routine clinical care: effectiveness and moderators of outcome in a consecutive sample. Behaviour Research and Therapy 51, 742-752.

Ehlers A, Hackmann A, Grey N, Wild J, Liness S, Albert I, Deale A, Stott R, Clark DM (2014). A randomized controlled trial of 7-day intensive and standard weekly cognitive therapy for PTSD and emotion-focused supportive therapy. American Journal of Psychiatry 171, 294-304. 
Foa E, Hembree E, Rothbaum BO (2007). Prolonged Exposure Therapy for PTSD: Emotional Processing of Traumatic Experiences, Therapist Guide. Oxford: Oxford University Press.

Foa EB, Rothbaum BO (1998). Treating the trauma of rape. Cognitive Behavior Therapy for PTSD. New York: Guilford.

Grey N, McManus F, Hackmann A, Clark DM, Ehlers A (2009). Intensive cognitive therapy for post-traumatic stress disorder: case studies. In: A Casebook of Cognitive Therapy for Traumatic Stress Reactions (ed. N. Grey), pp. 111-130. Hove: Routledge.

Jones RSP, Banks R (2007). Behavioural treatment of PTSD in a person with Intellectual Disability. European Journal of Behaviour Analysis, 8, 251-256.

Murray H, Merritt C, Grey N (2015). Clients' experiences of returning to the trauma site during PTSD treatment: an exploratory study. Behavioural and Cognitive Psychotherapy 43, 1-11.

Tolin DF, Foa EB (1999). Treatment of a police officer with PTSD using prolonged exposure. Behavior Therapy 30, 527-538.

\section{Learning objectives}

After reading this article, people will be able to:

- Understand the theoretical rationale for conducting a site visit as part of cognitive therapy for PTSD.

- Consider, with their client, the most appropriate time in treatment to conduct a site visit.

- Assess potential risks associated with revisiting the scene of the trauma.

- Plan and carry out a site visit in a way which maximizes clinical benefit. 